This paper is published in the open archive of Mid Sweden University

DIVA http://miun.diva-portal.org

with permission of the publisher

Citation for the peer-reviewed published paper:

Edström P, Neuman M, Avramidis S, Andersson M. Geometry Related Inter-Instrument Differences in Spectrophotometric Measurements. Nordic Pulp \& Paper Research Journal. 2010;25(2):221-232.

URL to article at publishers site:

http://dx.doi.org/10.3183/NPPRJ-2010-25-02-p221-232 


\title{
Geometry Related Inter-Instrument Differences in Spectrophotometric Measurements
}

\author{
Per Edström, Magnus Neuman, Stefanos Avramidis and Mattias Andersson
}

KEYWORDS: Reflectance measurements, Spectrophotometry, Instrument geometry, Measurement differences, Radiative transfer, Anisotropy, Absorption, Transmittance

SUMMARY: The L\&W Elrepho $\mathrm{d} / 0$ and the Spectrolino $45 / 0$ instruments are examined using paper samples with different properties. External factors that influence the measurements - such as the sample background, the instrument calibration and the sample inhomogeneity are studied, and a methodology for their minimization is presented. Experimental measurements show that such external factors, if not minimized by proper routines, affect the inter-instrument differences far more (up to 4-5 $\Delta E^{*}{ }_{a b}$ ) than the instrument geometry (the effect of which is small and of order $0.1 \Delta E^{*}{ }_{a b}$ ). The DORT2002 radiative transfer model is used to simulate differences caused by instrument geometry. The simulated and measured differences are found to agree in magnitude, and the differences are mapped against sample properties. It is observed that the $45 / 0$ instrument detects higher reflectance from paper samples with negligible absorption and transmittance. When there is considerable absorption (dyed samples) or transmittance (thin samples), the $\mathrm{d} / 0$ instrument detects higher reflectance. The physical mechanism behind this behavior is studied and explained using DORT2002, and the instrument differences are shown to depend on the anisotropy of the reflected light. The model/measurement agreement is satisfactory as the characteristic behavior is captured in almost all cases studied. This new understanding is important for facilitating accurate data exchange between the paper and graphic arts industries, but also for interpretation of reflectance measurements in general.

\section{ADDRESSES OF THE AUTHORS: Per Edström (per.edstrom@miun.se), Magnus Neuman (magnus.neuman@miun.se) and Mattias \\ Andersson (mattias.andersson@miun.se): Mid Sweden University, Department of Natural \\ Sciences, Engineering and Mathematics, SE-871 88 Härnösand, Sweden. Stefanos Avramidis (savramidis@fastmail.net): KTH - Royal Institute of Technology, Department of Numerical Analysis, SE-100 44 Stockholm, Sweden. \\ Corresponding author: Magnus Neuman}

The paper and the graphic arts industries use different spectrophotometer instruments to assess the optical properties of printed and unprinted paper through reflectance measurements. The instruments differ significantly in geometrical characteristics since the illumination and detection conditions are not the same (ISO 2469, ISO 5-4, ISO 13655, DIN 5033), and the different instrument types have been reported to yield incomparable measurements (Radencic et al. 2008; Seghi 1990). Explanations have been proposed (Spooner 1994) based on differences in spectrophotometer properties like detector linearity, spectral calibration, spectral bandwidth treatment, translucence phenomena and other edge effects.

The standardized instruments for measuring reflectance used in the paper and graphic arts industries have a d/0 or $45 / 0$ geometry, respectively. The $\mathrm{d} / 0$ geometry, used in the paper industry, illuminates the paper sample diffusely and measures reflectance in $0^{\circ}$, i.e. in the normal direction. The $45 / 0$ geometry, used in the graphic arts industry, illuminates the sample with directed light incident in $45^{\circ}$ from the normal to the paper and measures reflectance in $0^{\circ}$. Apart from the geometrical differences, the instruments differ in calibration routines, sample background, measurement spot size and UV content of the illumination. Furthermore, there exist few if any standardized methods for communication of optical properties between the paper and graphic arts industries. Independently of each other, they have developed standardized methods for the measurement of optical properties, optimized for the different requirements and purposes in each industry. The differences between the color measurement methods used in the two industries do inarguably cause problems in both color management and proofing. Fluorescence is by far the biggest problem, and steps have been taken to address this in 45/0 instruments with adjustable UV content.

The optical model in most frequent use in the industry, the Kubelka-Munk (KM) model (Kubelka, Munk 1931; Kubelka 1948), considers light travelling in the two main directions up and down, and the medium is characterized by its phenomenological KM scattering and absorption coefficients $s$ and $k$. However, the KM model does not allow for investigation of the influence of the angle resolved intensity, and thereby of the instrument geometry, on the detected reflectance factor, $R$.

To accurately model the instruments' geometry it is convenient to use radiative transfer theory (Chandrasekhar 1960), where the medium is 
characterized by its physically objective scattering and absorption parameters (cross sections) $\sigma_{s}$ and $\sigma_{a}$. For a medium with an isotropic single-scattering process, there are simple approximate relations (exact relations do not exist (Neuman, Edström 2010a)) between the sets of scattering and absorption parameters, $s=\frac{3}{4} \sigma_{s}$ and $k=2 \sigma_{a}$. The relation between scattering and absorption is often indicated using the dimensionless single scattering albedo, $a=\sigma_{s} /\left(\sigma_{s}+\sigma_{a}\right)$. Radiative transfer theory describes in detail the interaction of light and turbid media, such as paper, and it allows for the calculation of the angle resolved intensity of light. In this way the geometrical differences in illumination and detection conditions can be included in the model and simulated. The KM model has been shown to be a simplified case of the more general radiative transfer theory (Edström 2004, Neuman, Edström 2010a, Neuman, Edström 2010b). Edström (2005) has developed the computational tool DORT2002 ${ }^{1}$ for numerical solution of the radiative transfer problem, and this tool has been thoroughly evaluated (Edström 2008; Edström 2009). This tool is adapted to applications in the paper industry and it is based on the same assumptions about the paper medium as KubelkaMunk theory uses (a continuum of scattering and absorption sites), but it is generalized to handle angular variations in the light. Pierce and Marcus (1996) have presented a comparison of KM and radiative transfer models for $45 / 0$ and $\mathrm{d} / 0$ measurements, but their focus was on the differences between the two theoretical models and not on the influence of instrument geometry.

The purpose of the present work is to investigate the inter-instrument differences between instruments of $d / 0$ and $45 / 0$ type, and to examine to what extent they can be explained by taking the instrument geometry into account. This work also aims to quantify these differences, and to suggest what can be done to minimize them. It is the purpose to show how both proper measurement routines and better measurement data interpretation can facilitate this, and to show the size of the errors made if this is not considered.

\section{Methods and Materials}

Paper samples were produced and measured, and the measurements were simulated as described in separate sections below. The difference in the detected reflectance factor from $\mathrm{d} / 0$ and $45 / 0$ spectrophotometers was measured and simulated for a wide variety of different paper samples. A measure of the reflectance factor difference was introduced as $\Delta R=R_{45 / 0}-R_{d / 0}$, therefore a positive $\Delta R$ means that the $45 / 0$ detects larger reflectance factor than the $d / 0$ and vice versa. This was used to quantify the differences between instrument types, and both theoretical and experimental arguments were used to explain the findings.

\section{Paper samples}

To study the effect of scattering, absorption and paper thickness on the differences between the two types of instruments, a series of samples with low grammage and varying filler and dye content was prepared with a Formette Dynamique. All samples were made from a mix of equal amounts of kraft and birch pulps, having Shopper/Riegler numbers $18^{\circ}$ and $23^{\circ} \mathrm{SR}$ respectively. Sheets of approximate grammage of $30 \mathrm{~g} / \mathrm{m}^{2}$ were prepared, each with and without filler (PCC SYNCARB FO 474-MJ). All sheets contain $0.02 \%$ PAM, 0.2\% Bentonit and $0.35 \%$ Cerestar. The amount of blue dye was changed from 0 to $1 \%$ of the fiber weight in four steps, which gives 8 types of sheets in total. The dye used was Levacell Fast Blue KS-6GLL Liquid, manufactured by Lanxess. This is a cationic direct dye and it is assumed that it does not affect the structure of the paper. The sheets were dried in a cylinder dryer for 5 minutes at a temperature of $105^{\circ} \mathrm{C}$ and a pressure of 1.50 bars. The samples do not contain any FWA. These samples are denoted Series 1 and their properties are shown in Table 1.

Table 1. Series 1 consists of eight samples with grammage 30 $\mathrm{g} / \mathrm{m}^{2}$ that vary in amount of dye (\% of fiber weight) and filler.

\begin{tabular}{ll}
\hline Four samples, no fillers & Four samples, 22 \% fillers \\
\hline $0.00 \%$ dye content & $0.00 \%$ dye content \\
$0.33 \%$ blue dye content & $0.33 \%$ blue dye content \\
$0.67 \%$ blue dye content & $0.67 \%$ blue dye content \\
$1.00 \%$ blue dye content & $1.00 \%$ blue dye content \\
\hline
\end{tabular}

The spectral variation of scattering and absorption parameters for one of the samples in Series 1 is illustrated in Fig 1. The similarity between the phenomenological KM parameters $s$ and $k$ and the objective parameters $\sigma_{s}$ and $\sigma_{a}$ of radiative transfer theory is evident.

A second series of highly scattering samples was also used in this work. Three different filler contents and three different grammages were chosen and prepared with a small-scale experimental paper machine. All samples were made from a mix of $25 \%$ long fiber and $75 \%$ short fiber pulps, both having Shopper/Riegler numbers $25^{\circ} \mathrm{SR}$. Sheets of approximate grammage 80,100 and $120 \mathrm{~g} / \mathrm{m}^{2}$ were

${ }^{1}$ DORT2002 is freely available from the authors 


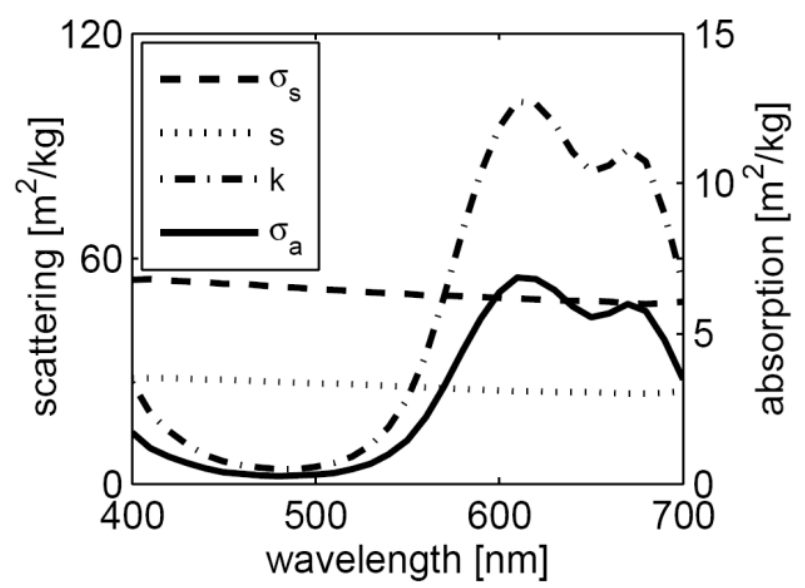

Fig 1. Comparison of the physically objective scattering and absorption parameters $\sigma_{\mathrm{s}}$ and $\sigma_{\mathrm{a}}$ of radiative transfer theory and the phenomenological KM parameters $s$ and $k$, for the $30 \mathrm{~g} / \mathrm{m}^{2}$ sample containing $0.33 \%$ dye and no fillers from Table 1. The parameter sets are obtained by using DORT2002 and KM respectively on standardized paper industry reflectance factor measurements. The similarity of the two parameter sets is evident.

prepared, each with filler contents of approximately $0 \%, 15 \%$ and $30 \%$ PCC. All sheets contain $0.05 \%$ Bentonit SH, $0.01 \%$ Percol 178 and $0.19-0.24 \%$ C*Bond HR35844 together with 0.13-0.17\% Kemsize 200. The samples do not contain any dye or FWA. These samples are denoted Series 2 and their properties are shown in Table 2.

Table 2. Series 2 consists of nine samples that vary in grammage and amount of filler. No samples contain dye.

\begin{tabular}{ccc}
\hline $\begin{array}{c}\text { Three samples, no } \\
\text { fillers }\end{array}$ & $\begin{array}{l}\text { Three samples, } \\
15 \% \text { fillers }\end{array}$ & $\begin{array}{l}\text { Three samples, } \\
\mathbf{3 0} \% \text { fillers }\end{array}$ \\
\hline $80 \mathrm{~g} / \mathrm{m}^{2}$ & $80 \mathrm{~g} / \mathrm{m}^{2}$ & $80 \mathrm{~g} / \mathrm{m}^{2}$ \\
$100 \mathrm{~g} / \mathrm{m}^{2}$ & $100 \mathrm{~g} / \mathrm{m}^{2}$ & $100 \mathrm{~g} / \mathrm{m}^{2}$ \\
$120 \mathrm{~g} / \mathrm{m}^{2}$ & $120 \mathrm{~g} / \mathrm{m}^{2}$ & $120 \mathrm{~g} / \mathrm{m}^{2}$ \\
\hline
\end{tabular}

Fluorescence and surface characteristics influence strongly the reflectance measurements, and introduce differences between the $\mathrm{d} / 0$ and $45 / 0$ instruments that are far more significant than the geometrical differences studied in this work. It has been shown (Andersson, Norberg 2007) that fluorescence can introduce errors up to $7 \Delta E_{a b}^{*}$. This is, among others, due to the different treatment of UV (illumination, calibration and filtering) between the instruments. The interested reader can find more on this in Radencic et al. (2008).

Samples included in this work were uncalendared uncoated papers with low gloss and no added FWA. Gloss measurements were made with a Zehntner glossmeter. Ten measurements were made on each sample, and the gloss was found to be low with an average of 1.0 for $20^{\circ}$ and 2.9 for $60^{\circ}$ and $75^{\circ}$. The surface roughness averaged at 250 Bendtsen for the samples with high filler content, and at 550 Bendtsen for the samples with no fillers.

\section{Measurements}

The $d / 0$ instrument used in this work was a L\&W Elrepho, which complies with ISO 2469 (1994). It is calibrated with specific reference samples in accordance with the ISO hierarchy. The illumination is diffuse, using an integrating sphere with a gloss trap. The detection uses a $4^{\circ}$ aperture sensor directed along the normal to the sample, and requires a sample area of $34 \mathrm{~mm}$ radius. The illuminated area is larger than the measured area, which avoids edge effects. Its repeatability is given by the manufacturer to be $0.01 \Delta E^{*}$, where the CIE 1976 color difference in the $\mathrm{L}^{*} \mathrm{a}^{*} \mathrm{~b}^{*}$ color space is given by:

$$
\Delta E_{a b}^{*}=\sqrt{\left(\Delta L^{*}\right)^{2}+\left(\Delta a^{*}\right)^{2}+\left(\Delta b^{*}\right)^{2}}
$$

(Wyzecki, Stiles 2000).

Instruments with $45 / 0$ geometry have different characteristics depending on the manufacturer. The $45 / 0$ instrument used in this work was a GretagMacbeth Spectrolino, which complies with DIN 5033 (1992). It is calibrated using tiles provided by the manufacturer for each specific instrument. The illumination is of ring-type at $45^{\circ}$ to the normal to the sample. The detection uses a $4^{\circ}$ aperture sensor directed along the normal to the sample, and requires a sample area of $4 \mathrm{~mm}$ radius. The illuminated area is equal to the measured area, which means there will be some edge effects. Its repeatability is given by the manufacturer to be $0.03 \Delta E_{a b}^{*}$.

The samples in Series 1 and 2 were measured in these $d / 0$ and $45 / 0$ instruments. Reflectance factor values were recorded at every $10 \mathrm{~nm}$ in the wavelength range of the respective instrument, and they were used to calculate measured interinstrument differences and for comparison with simulations. All conversions from measured reflectance spectra to $\mathrm{L}^{*} \mathrm{a} \mathrm{b}^{*}$ were done using the color weighting functions for D65 illuminant and $10^{\circ}$ observer.

Several external factors can influence the measurements. The most important are the measurement background, the instrument calibration and the inhomogeneity of the paper sample. Special care has to be taken to minimize these factors. This facilitates the isolation of the effects of the instrument geometry for the present study, but it also illustrates the error made when not recognizing and minimizing these external factors. The following three points were employed in this work. 
- A highly absorbing black matte surface was used as background - the same in both instruments ${ }^{2}$. The measured reflectance factor for the background is about 0.05 , and the spectral variation is shown in Fig 2.

- The calibration of the instruments was done with the instrument-specific tiles and routines supplied by the manufacturer (the $45 / 0$ devices must unfortunately be calibrated individually - a tile from another instrument of the same type from the same manufacturer gives miscalibration).

- A set of five measurements over different random spots was averaged to reduce the effect of the sample inhomogeneity.

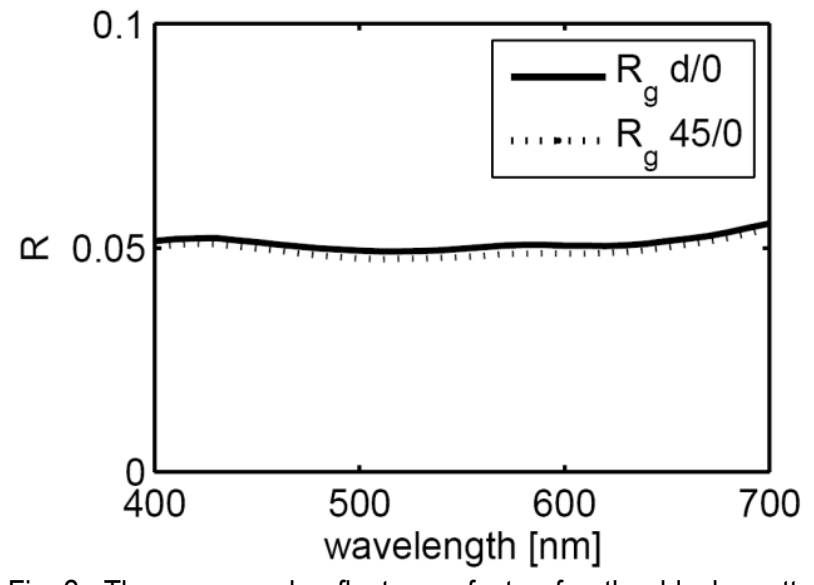

Fig 2. The measured reflectance factor for the black matte surface used as common background in the $d / 0$ and $45 / 0$ reflectance measurements.

In addition to this, the different instruments treat UV differently (illumination, calibration and filtering), but such effects were avoided in this work by using non-fluorescent samples.

\section{Simulations}

The radiative transfer based light scattering simulation tool DORT2002 (Edström 2005) was set up to simulate the standardized $\mathrm{d} / 0$ and $45 / 0$ instruments by modeling their geometrical illumination and detection conditions. This made it possible to make theoretical predictions about the

\footnotetext{
2 The $\mathrm{d} / 0$ instrument uses, as specified by paper industry standard, a black cavity as background when measuring a single sheet. The 45/0 instrument is delivered with a white and a black background surface. A highly absorbing black matte surface was used in both instruments when measuring a single sheet in order to have an identical background with minimum reflectance. The reflectance factor so recorded is for convenience denoted $R_{0}$ in this paper for both instrument types, although this symbol denotes measurement over a black cavity according to paper industry standards.
}

inter-instrument differences, which can then be compared to the corresponding measurements.

The detected reflectance factor for the $\mathrm{d} / 0$ and $45 / 0$ instruments was simulated for a range of varying scattering and absorption properties to cover a reasonable spectrum of relevant paper samples translucent and opaque, with and without dye. The scattering coefficient $\sigma_{s}$ was spanned from 0 to $200 \mathrm{~m}^{2} / \mathrm{kg}$ and the absorption coefficient $\sigma_{a}$ from 0 to $30 \mathrm{~m}^{2} / \mathrm{kg}$ (this approximately corresponds to $s$ in the range 0 to $150 \mathrm{~m}^{2} / \mathrm{kg}$, and $k$ in the range 0 to $60 \mathrm{~m}^{2} / \mathrm{kg}$ ). The grammage $w$ was set to $30 \mathrm{~g} / \mathrm{m}^{2}$ and $80 \mathrm{~g} / \mathrm{m}^{2}$, and isotropic single scattering was used.

The simulated reflectance factors were used to calculate simulated inter-instrument differences, and for comparison with the measured ones from samples in Series 1 and 2.

\section{Results}

\section{Simulated inter-instrument differences}

Fig 3 shows the simulated difference in $R_{0}$, i.e. the reflectance factor from a single sheet (left), and the simulated difference in $R_{\infty}$, i.e. reflectance factor from an opaque pad (right). The simulations are made for a range of scattering and absorption coefficients for $30 \mathrm{~g} / \mathrm{m}^{2}$ and $80 \mathrm{~g} / \mathrm{m}^{2}$ samples. A single point on the surface thus corresponds to one specific wavelength with those scattering and absorption properties. The inter-instrument differences predicted by the model are clearly of small magnitude; the maximum difference is approximately 0.02 reflectance factor units.

Based on Fig 3, several features can be distinguished:

- With high absorption and moderate scattering (I), an instrument with $\mathrm{d} / 0$ geometry is predicted to detect higher reflectance factor than an instrument with $45 / 0$ geometry. This parameter region corresponds to dyed papers.

- For samples with very low scattering and moderate to high absorption, i.e. dark samples (II), the $\mathrm{d} / 0$ instrument is predicted to detect higher reflectance factor than the $45 / 0$ instrument.

- For very light samples (III), the $45 / 0$ instrument is predicted to detect higher reflectance factor. This is the region with the largest difference between the two instrument geometries. The difference is larger for higher scattering values.

- The parameter region around minimal absorption and scattering (IV) corresponds to an emulsion or air spray, rather than paper samples, and is thus not relevant in paper applications. 


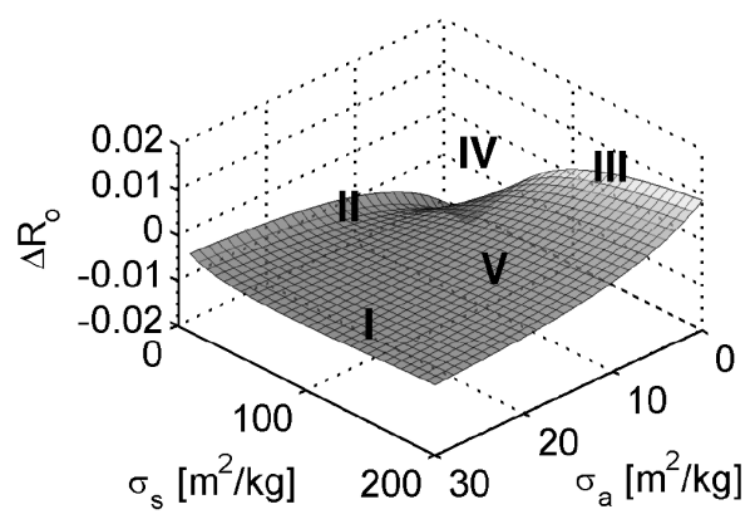

(a)

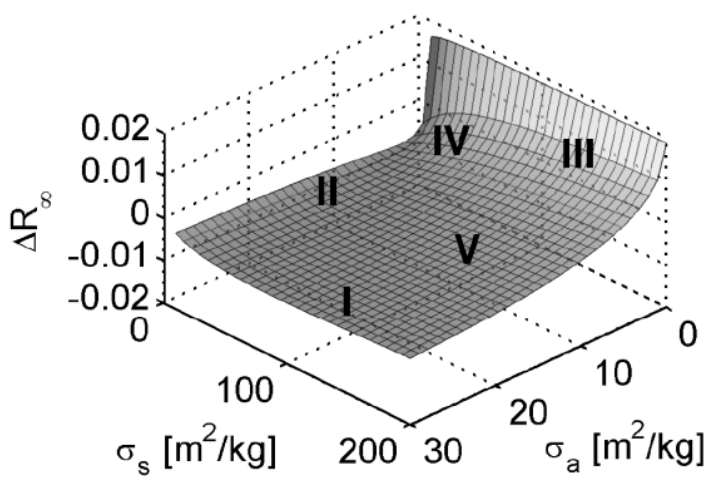

(b)

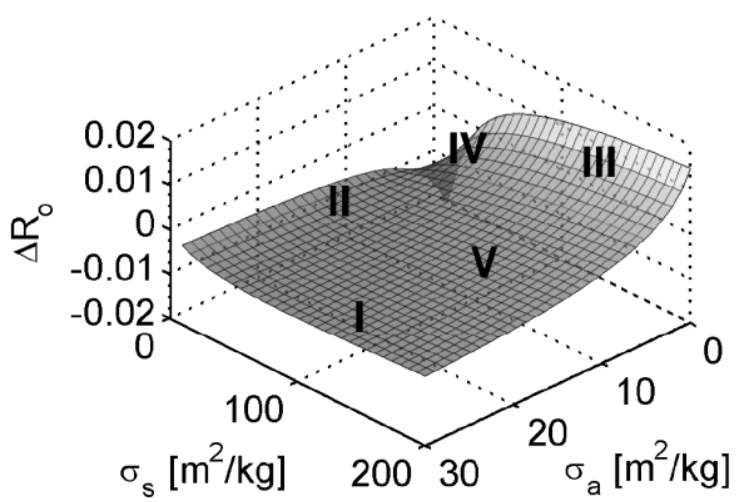

(c)

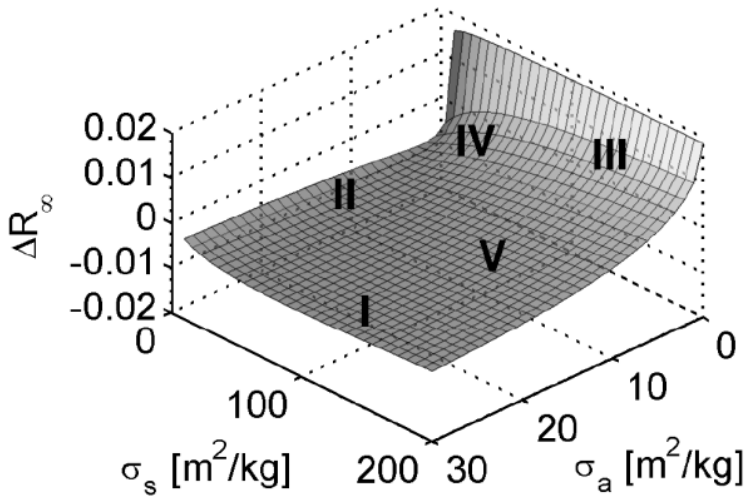

(d)

Fig 3. The simulated reflectance differences $\Delta R_{0}$ (left) and $\Delta R_{\infty}$ (right) plotted against the range of scattering and absorption coefficients for $30 \mathrm{~g} / \mathrm{m}^{2}$ (upper) and $80 \mathrm{~g} / \mathrm{m}^{2}$ (lower) samples. The middle area ( $\mathrm{V}$ - corresponding to lightly dyed samples) is where inter-instrument differences are small. The area of low absorption (III - corresponding to white paper) is where $45 / 0$ measures larger $R$ than $\mathrm{d} / 0$, and the rest of the area (I and II - mostly corresponding to dyed or printed paper) is where $\mathrm{d} / 0$ measures larger $R$ than $45 / 0$. The inter-instrument differences $\Delta R$ are clearly small - below 0.02 reflectance factor units.

- The large flat region (V) corresponds to lightly dyed papers. In this region, the inter-instrument difference is very small.

- High transmittance tends to decrease the interinstrument difference in the case of low absorption since then $\Delta R_{0}<\Delta R_{\infty}$. Very light paper samples with low grammage are thus predicted to result in small inter-instrument differences.

The simulations predict that white plain paper would have the largest inter-instrument differences, and that instruments with $45 / 0$ geometry would detect higher reflectance factor for this type of paper, but that the differences would be smaller for low grammages. As the absorption is increased, the $\mathrm{d} / 0$ would eventually detect a higher reflectance factor. This means that white and dyed paper exhibit significantly different characteristics in this respect.

\section{Measurements}

The measured inter-instrument differences are small, as can be seen in Fig 4, and the dyed samples have smaller differences than the non-dyed ones. Both size and sign of the inter-instrument differences are in agreement with the simulated model predictions, as are the variations with sample characteristics.

Since the inter-instrument differences are so small (it should be noted that they are close to instrument precision, so some irregular variations are likely to be present), they can be better observed by plotting the reflectance factor difference $\Delta R=R_{45 / 0}-R_{d / 0}$. In Fig $5, \Delta R$ is presented for the non-dyed samples of $80 \mathrm{~g} / \mathrm{m}^{2}$ and $120 \mathrm{~g} / \mathrm{m}^{2}$. For these samples, the color difference reaches $0.2 \Delta E^{*}$ ab. The presence of fillers increases the differences for samples of higher grammage. 


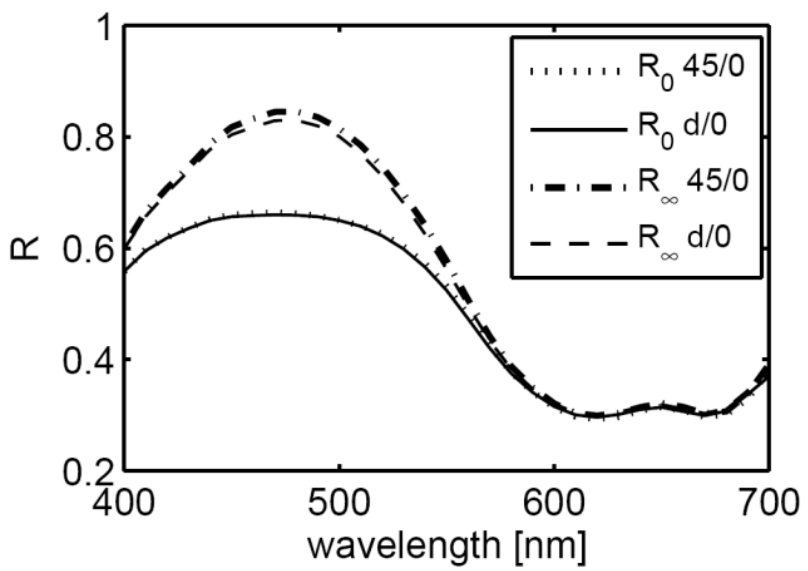

(a)

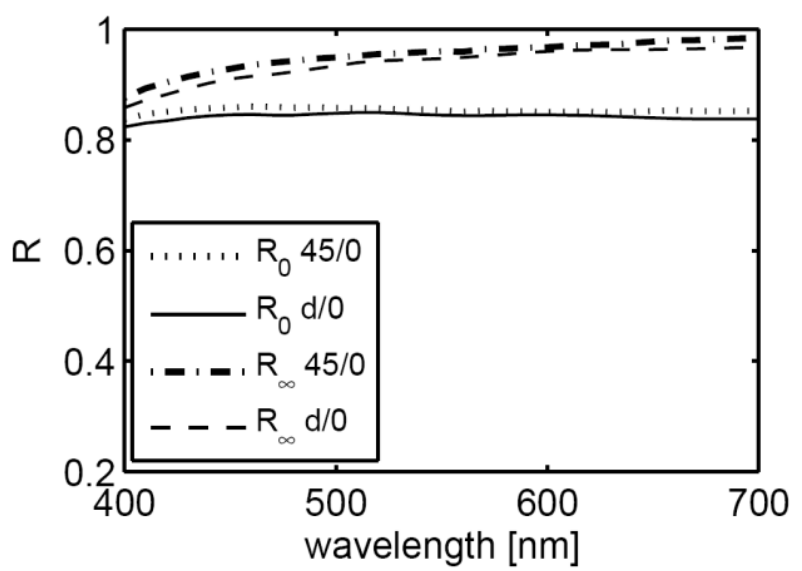

(b)

Fig 4. Spectral $R_{0}$ and $R_{\infty}$ reflectance factors are plotted for both instrument types for a thin blue dyed sample (a) (Series $1,30 \mathrm{~g} / \mathrm{m}^{2}$, $1 \%$ dye, $22 \%$ fillers), and for a non-dyed sample (b) (Series 2, $80 \mathrm{~g} / \mathrm{m}^{2}$, no dye, $30 \%$ fillers). The inter-instrument differences are small, but they are larger for the non-dyed sample. This is in accordance with the predictions from the model simulations.

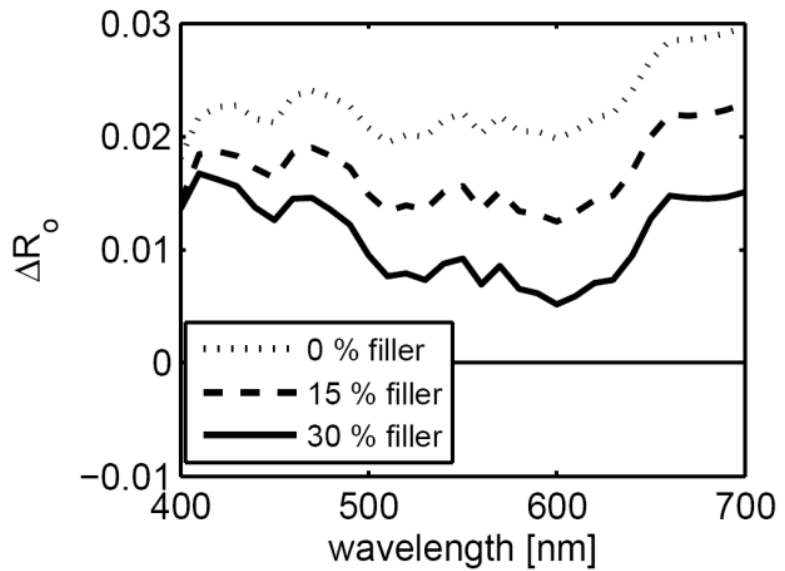

(a)

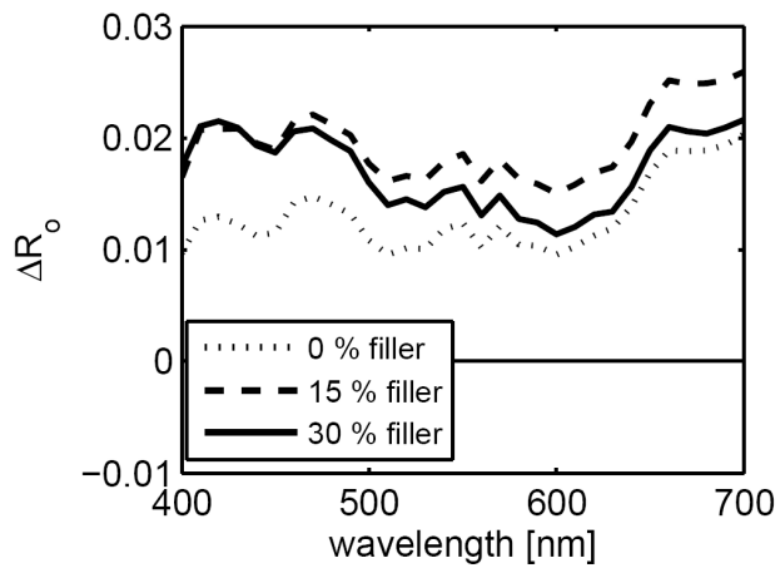

(c)

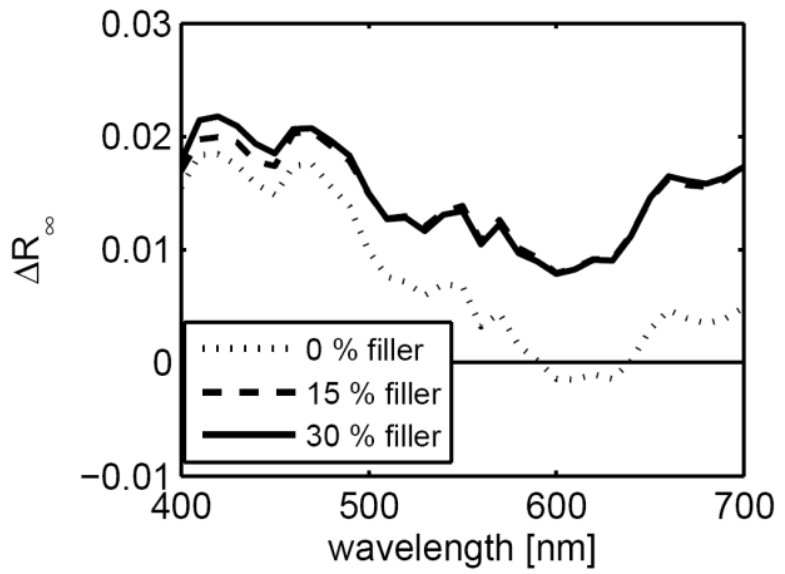

(b)

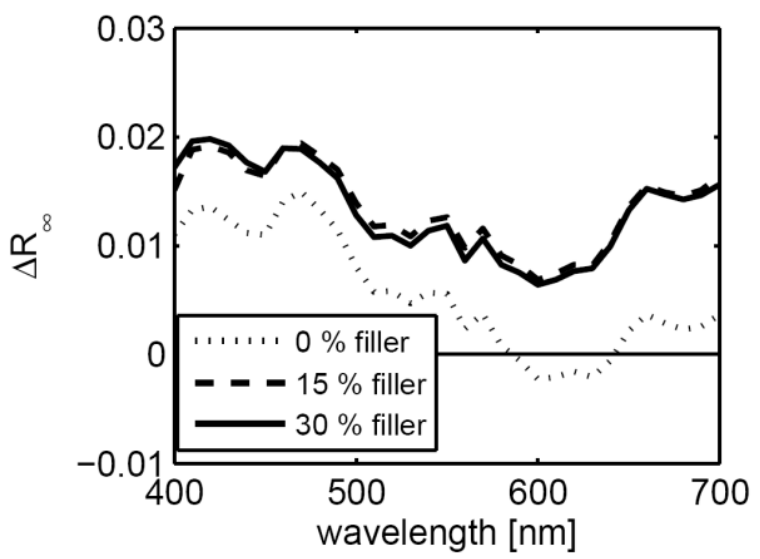

(d)

Fig 5. Measured $\Delta R_{0}$ (left) and $\Delta R_{\infty}$ (right) for non-dyed samples of grammages $80 \mathrm{~g} / \mathrm{m}^{2}$ (upper) and $120 \mathrm{~g} / \mathrm{m}^{2}$ (lower) and three different filler contents from Series 2 . The inter-instrument differences are small, and generally positive.

For the samples of lower grammage, this effect is not present due to influence of transmittance ${ }^{3}$. The

\footnotetext{
3 In fact, the inhomogeneity of samples of lower grammage and low filler content makes the deviation between the small measurement spots of the $45 / 0$ instrument have the same size as the entire inter-
}

inter-instrument differences are small, generally positive (larger values for 45/0) and approximately

instrument difference - what is seen in Fig 5 (a) is therefore mostly the different degree of background influence on different spots, and not instrument differences. 


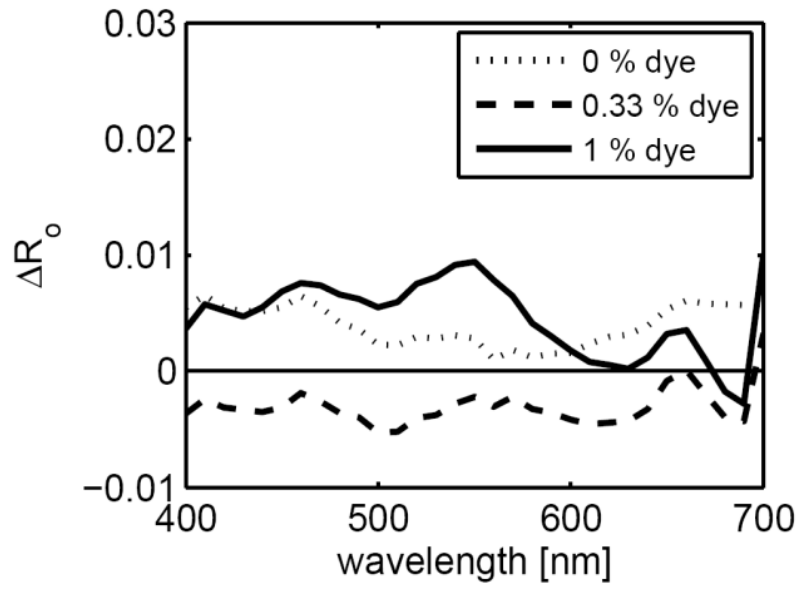

(a)

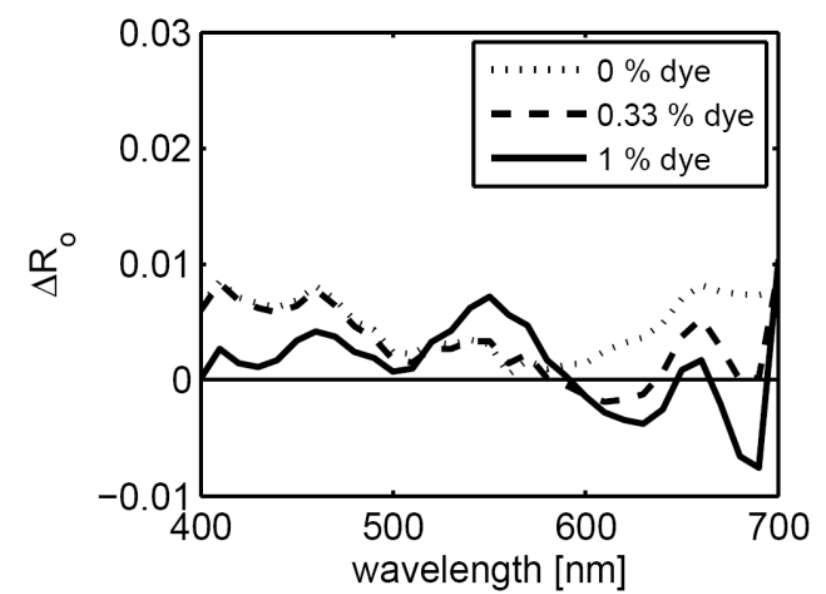

(c)

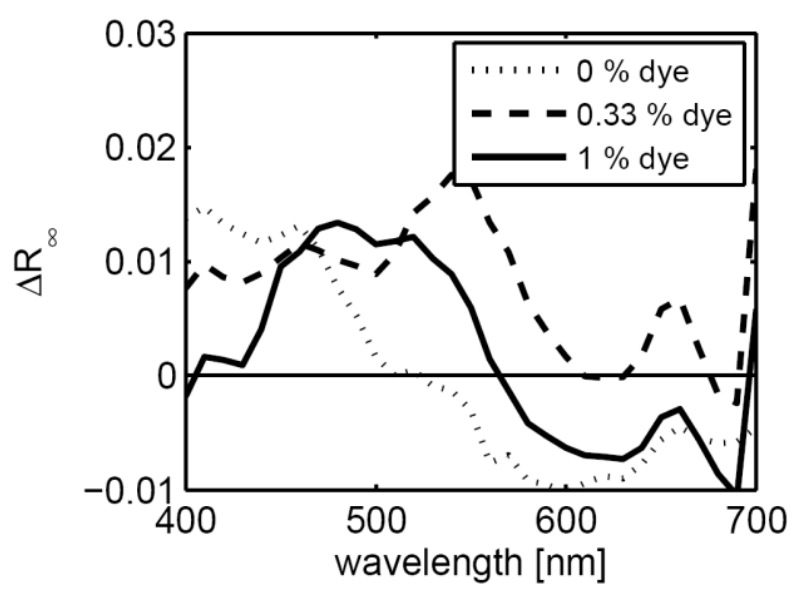

(b)

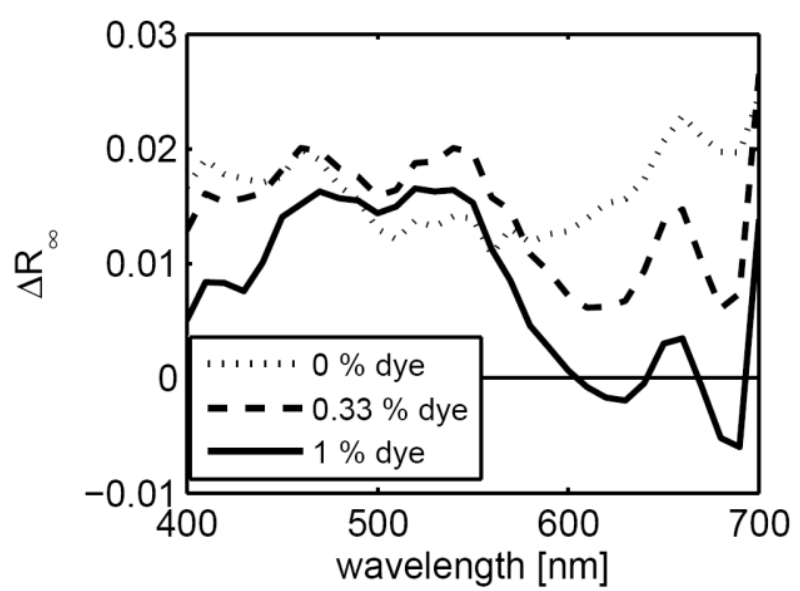

(d)

Fig 6. Measured $\Delta R_{0}$ (left) and $\Delta R_{\infty}$ (right) for dyed samples with (lower) and without (upper) fillers and for three different dye contents from Series 1 . The inter-instrument differences are small, and mostly positive. The regions of high dye absorption generally show negative $\Delta R$. The $\Delta R_{\infty}$ of these low grammage samples is higher in general than the respective $\Delta R_{0}$.

constant with only small variations over the wavelength range. This is in accordance with the model predictions. Unexpectedly, the $\Delta R_{\infty}$ difference of the samples decreases in the red wavelength region, and in some cases $\Delta R_{\infty}$ is negative.

In Fig 6, the measured $\Delta R$ is shown for samples with varying amount of blue dye. $\Delta R$ is generally small, very close to the instrument precision. For the wavelengths corresponding to low absorption of the dye, all samples have $\Delta R>0(45 / 0$ detecting larger values). The dyed samples show similar characteristics with each other, with $\Delta R<0$ for wavelengths corresponding to high absorption of the dye. The differences are larger in the $R_{\infty}$ measurements than in $R_{0}$ for these samples of low thickness. This is all in agreement with the model predictions.

The $\Delta E^{\star} a b$ measure of color difference can also be utilized for illustrating the inter-instrument differences with a single, aggregate value. It should be noted, however, that since this is a weighted spectral average, it gives a measure that may conceal some spectral aspects. In Tables 3 and 4, thin dyed samples exhibit the smallest differences overall in the $R_{0}$ measurement. The same samples also show $\Delta R_{0}<\Delta R_{\infty}$. Tables 3 and 4 also show that $\Delta R_{0}$ is larger for the undyed samples of higher grammage than for the dyed samples of lower grammage. All these experimental findings are in accordance with the model predictions above.

Table 3. $\Delta E^{\star}{ }_{a b}$ differences of Spectrolino from L\&W Elrepho of sample Series $1\left(30 \mathrm{~g} / \mathrm{m}^{2}\right)$.

\begin{tabular}{|c|c|c|c|c|}
\hline & \multicolumn{2}{|c|}{$\boldsymbol{R}_{0}$} & \multicolumn{2}{|c|}{$\boldsymbol{R}_{\infty}$} \\
\hline & $0 \% \mathrm{f}$ & $22 \% f$ & $0 \% f$ & $22 \% f$ \\
\hline $0 \% \mathrm{~d}$ & 0.05 & 0.06 & 0.23 & 0.14 \\
\hline $0.33 \% \mathrm{~d}$ & 0.03 & 0.07 & 0.16 & 0.17 \\
\hline $0.67 \% \mathrm{~d}$ & 0.04 & 0.03 & 0.21 & 0.18 \\
\hline $1 \% \mathrm{~d}$ & 0.08 & 0.06 & 0.19 & 0.19 \\
\hline
\end{tabular}

Table 4. $\Delta E^{*}$ ab differences of Spectrolino from L\&W Elrepho of sample Series 2 (0\% dye)

\begin{tabular}{lrrrrrr}
\hline W, g/m & \multicolumn{3}{c}{$\boldsymbol{R}_{\boldsymbol{0}}$} & & \multicolumn{3}{c}{$\boldsymbol{R}_{\infty}$} \\
\hline & $\mathbf{0 \%}$ f & $\mathbf{1 5 \%} \mathbf{f}$ & $\mathbf{3 0 \%} \mathbf{f}$ & $\mathbf{0 \%} \mathbf{f}$ & $\mathbf{1 5 \%} \mathbf{f}$ & $\mathbf{3 0 \%} \mathbf{f}$ \\
$\mathbf{8 0}$ & 0.19 & 0.14 & 0.11 & 0.18 & 0.15 & 0.16 \\
$\mathbf{1 0 0}$ & 0.18 & 0.13 & 0.17 & 0.17 & 0.13 & 0.15 \\
$\mathbf{1 2 0}$ & 0.10 & 0.16 & 0.15 & 0.14 & 0.14 & 0.14 \\
\hline
\end{tabular}


In general, the measured inter-instrument differences are in agreement with the model predictions. The measurement results can be summarized as:

- Dyed paper samples have $\Delta R>0$ (45/0 detecting larger values) in wavelengths corresponding to low absorption of the dye, and $\Delta R<0$ (d/0 detecting larger values) for wavelengths corresponding to high absorption of the dye.

- Very light samples have $\Delta R>0$

- Samples without dye exhibit the largest instrument differences.

- For thin papers $\Delta R_{\infty}$ is larger than $\Delta R_{0}$.

- For low absorbing thick papers the interinstrument difference further increases with the presence of filler.

It should be noted for comparison that external factors, if not carefully minimized, affect the measurements to a much larger extent than the recorded inter-instrument difference caused by instrument geometry. This was tested by doing measurements without following the points described in the Methods section that were designed to minimize such influences. Using different background for the $R_{0}$ measurements caused differences up to $3.5 \Delta E^{*}{ }_{a b}$. Calibrating a $45 / 0$ instrument with a tile from another instrument of the same type caused differences up to $0.8 \Delta E^{*}{ }_{a b}$. Differences up to $0.3 \Delta E^{*}{ }_{a b}$ were obtained when carrying out five measurements on different spots/positions on the samples . In addition to this, it has been shown (Andersson, Norberg 2007) that fluorescence can introduce errors up to $7 \Delta E^{*}{ }_{a b}$, which is far more significant than the geometrical differences studied in this work. The interested reader can find more on this in Radencic et al. (2008). All these factors were, as mentioned above, minimized in the procedure used in this work.

\section{Explaining geometry related inter-instrument differences}

Neuman and Edström (2010a, 2010b) explain in some detail what causes anisotropy in the light reflected from turbid media such as paper. The conclusions are based on the fact that the angular distribution of the reflected light depends on the relative contributions to the reflectance from different depths in the medium. If near-surface bulk scattering dominates, relatively more light is reflected in large polar angles, i.e. in angles close to the medium surface. If light reflected from larger depths in the medium dominates the reflectance, relatively more light is reflected in the normal direction of the paper. Consequently, a series of factors applicable to paper can be distinguished:
1. Thin samples are dominated by near-surface bulk scattering since light penetrating the medium further has a high probability of being transmitted. These samples thus reflect more light in angles close to the paper surface.

2. Highly absorbing samples are dominated by near-surface bulk scattering since light penetrating the medium further has a high probability of being absorbed. These samples thus also reflect more light in angles close to the paper surface.

3. Illumination with more light incident in angles close to the medium surface results in the domination of near-surface bulk scattering since the light has to travel larger distance to reach larger depths and thus has a higher probability of being absorbed. This leads to more light being reflected in angles close to the surface.

4. Normally incident illumination results in more light scattering deeper inside the medium since the path to reach a certain depth is shorter with this illumination. This leads to more light being reflected close to the normal direction of the sample.

Every situation or measurement setup constitutes a combination of these four factors and the angular distribution of the reflected light will depend on the relative influence of each of them.

The probability $P$ of directed light incident in polar angle $\theta^{\prime}$ to reach a depth larger than $b$ in a medium with extinction coefficient $\sigma_{e}$ is :

$P(x \geq b)=\int_{b / \cos \theta^{\prime}}^{\infty} \sigma_{e} \exp \left(-\sigma_{e} x^{\prime}\right) d x^{\prime}=\exp \left(-\sigma_{e} b / \cos \theta^{\prime}\right)$

The characteristic depth where the light intensity is half the intensity of the incident light can be expressed as $b^{50 \%}=\ln (2) \cos \theta^{\prime} / \sigma_{e}$. Changing the angle of incidence $\theta^{\prime}$ of the directed illumination therefore changes this characteristic depth. If the illumination is not directed, such as the illumination of the $\mathrm{d} / 0$ instrument geometry or a perfectly diffuse illumination, the average of the cosine function over the polar angles of the illumination must be used. For the $\mathrm{d} / 0$ instrument with gloss trap, this becomes $\int_{\theta_{1}}^{\theta_{2}} \cos x d x /\left(\theta_{2}-\theta_{1}\right)=0.5637$, where the angles are the extension of the gloss trap, and the characteristic depth thus corresponds to a directed illumination incident at $55.7^{\circ}$. Since $45^{\circ}$ is smaller than this, it can be concluded that the characteristic depth $b^{50 \%}$ is larger for the $45 / 0$ geometry, and consequently that the incident light of this instrument geometry reaches relatively larger depths in a medium than the illumination of the $d / 0$ geometry. The reflected light in the $45 / 0$ geometry, part of which is detected by the instrument, is then 
dominated by light coming from larger depths in the paper compared to the $\mathrm{d} / 0$ geometry. The $\mathrm{d} / 0$ instrument geometry thus has a relatively larger part of near-surface bulk scattering compared to the $45 / 0$ geometry.

According to Neuman's and Edström's results described above, the angular distribution of the reflected light differs between the instruments, with more light thus being reflected in polar angles close to the paper surface in the $d / 0$ geometry. This is illustrated in Fig 7 where simulations of the BRDF (bidirectional reflectance distribution function) from a non-absorbing (albedo $a=1$ ) and non-transmitting medium with varying illumination is shown. The $\mathrm{BRDF}$ is proportional to the reflectance factor and the intensity of the reflected light (Nicodemus 1977).

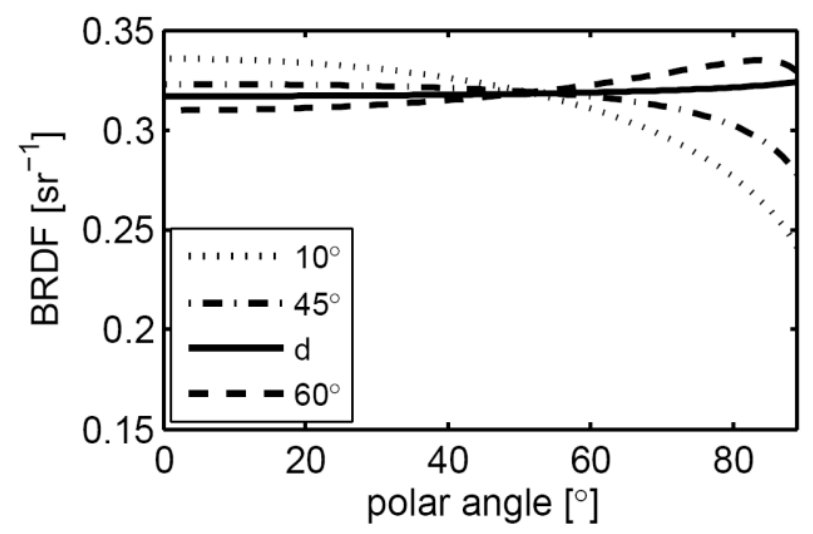

Fig 7. The BRDF of a non-absorbing and non-transmitting medium with varying illumination. A perfectly diffuse illumination would give a constant BRDF and thus an isotropic intensity of the reflected light. The plotted $\mathrm{d} / 0$ illumination (including gloss trap) gives close to isotropic intensity but slightly more light is reflected in large polar angles, which also holds for directed illumination from $60^{\circ}$. The $45 / 0$ illumination results in slightly more light being reflected around the normal direction, an effect that is even more pronounced for directed illumination from $10^{\circ}$ This is in accordance with expectations and model predictions.

Since the medium is non-absorbing and nontransmitting, the illumination angle is the only factor affecting the angular distribution of the reflected light. It can be seen that the angular dependence is in agreement with the reasoning above. The $d / 0$ illumination results in relatively more light being reflected in larger polar angles while the 45/0 illumination results in a higher reflectance around the normal direction. Since the detector of both instruments is placed in the normal direction, the $45 / 0$ instrument will detect a higher reflectance value in the case of a non-absorbing and nontransmitting sample. It is thus incorrect to interpret the detected reflectance as total or diffuse reflectance, or as independent of instrument geometry.
When absorption or transmittance increases, the relative amount of near-surface bulk scattering also increases. This means that factors 1 . and 2. above determine the angular distribution of the reflected light to a larger extent than factors 3. and 4. In Fig 8 (a) the simulated BRDF is shown for a highly absorbing and non-transmitting medium, and in Fig 8 (b) the BRDF is shown for a highly transmitting non-absorbing medium. It can be seen that the angular distribution of the reflected light is affected according to what is expected. That is, since factors 1. and 2. dominate, the relative amount of light reflected in large polar angles increases.

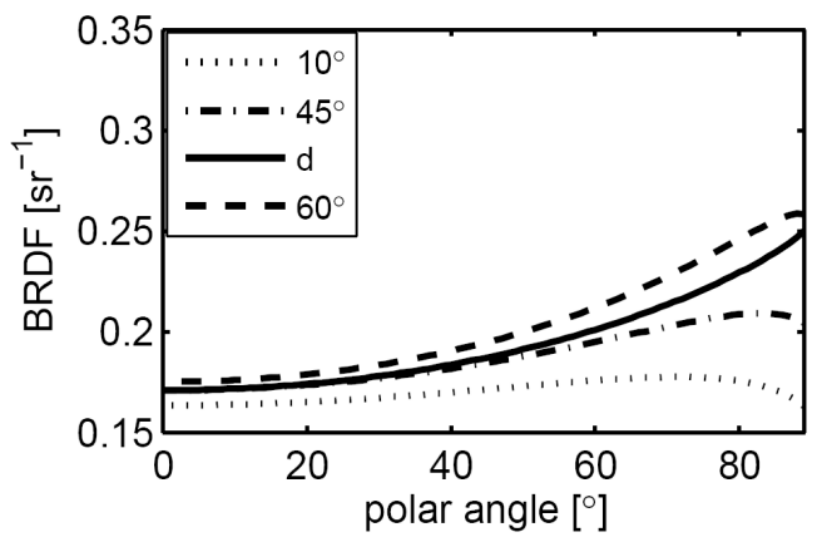

(a)

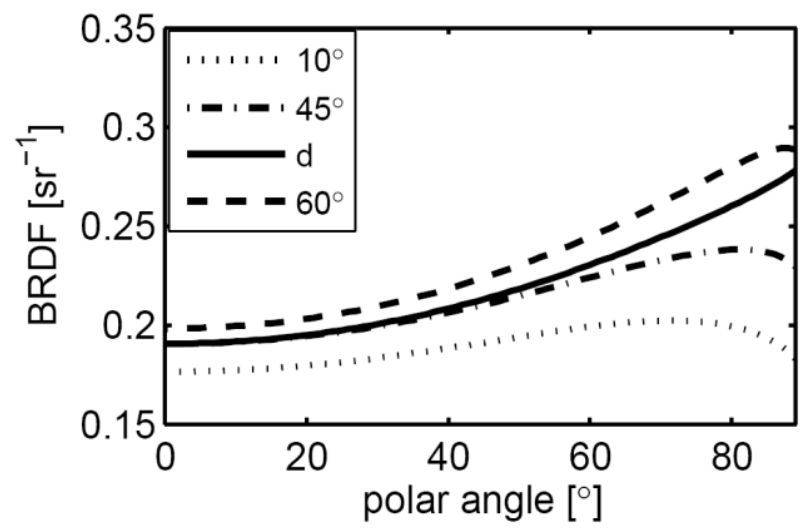

(b)

Fig 8. The BRDF of a highly absorbing (albedo a=0.95) nontransmitting medium (a) and a highly transmitting (grammage $w=0.01 \mathrm{~kg} / \mathrm{m}^{2}$ ) non-absorbing medium (b). High absorption causes the relative amount of near-surface bulk scattering to increase, and consequently the relative amount of light reflected in large polar angles increases irrespective of the illumination. Similarly, high transmittance also increases the relative amount of near-surface bulk scattering and relatively more light is reflected in large polar angles.

Fig 8 also shows that the BRDF is lower for all polar angles when the illumination is incident closer to the normal direction, i.e. when the illumination has a larger characteristic depth. This is because the larger characteristic depth results in a smaller probability of light exiting the medium at all, since the light is more likely to be transmitted or 
absorbed. Due to this fact, when there is sufficient amount of absorption or transmittance ( $a=0.95$ or $w=0.01 \mathrm{~kg} / \mathrm{m}^{2}$ ), the $\mathrm{d} / 0$ instrument illumination (including gloss trap) will detect a higher reflectance value than the $45 / 0$ instrument illumination for all polar angles, and consequently higher reflectance also at the detector location at $0^{\circ}$.

\section{Discussion}

The $d / 0$ and $45 / 0$ instrument geometries studied in this work vary in detection and illumination conditions. The angular distribution of the illumination differs significantly between these, and this causes the reflectance factor detected by the respective instrument to differ. This will in turn yield different color values or scattering and absorption parameters for identical samples, which is obviously not desired. The effects of instrument geometry when external factors are minimized can be explained.

External factors such as the sample background, the instrument calibration and the sample inhomogeneity affect the inter-instrument differences far more (up to $4-5 \Delta E^{*}{ }_{a b}$ ) than the instrument geometry (of order $0.1 \Delta E_{a b}^{*}$ ), but such effects can be minimized by proper routines. This is summarized in Table 5. Fluorescence can affect the interinstrument differences even more (up to $7 \Delta E^{*}{ }_{a b}$ ). This can currently only be eliminated by using samples with a minimum of fluorescence, and a general solution calls for standardization of the UV content of the illumination, of calibration routines, and of the use of various filters. The gloss, or specular reflections from the samples, is on the other hand inarguably a factor that firstly will affect the measurements, and secondly, cannot be eliminated. The uncoated samples used here, have rough surfaces and due to this, specular reflections will be included in the measurements. Furthermore, the surface reflection from uncoated paper is very complex, and will likely be handled differently by the two instruments due to the different geometries and designs of the gloss traps. However, in order to reduce the influence of gloss, the samples used here have low gloss values (with an average of $1.0-2.9$, see section on paper samples). Although gloss is present, it is reasonable to assume that its contribution is small compared to the bulk scattering. No polarization filters were used in the measurements.

There are obviously several color measurement instrumentation error mechanisms which are not considered in this study. Among these are detector linearity, spectral calibration, spectral bandwidth treatment, translucence phenomena and other edge effects. These, and many others are more thoroughly described elsewhere (Spooner 1994, Berns 2000). Moreover, it should be noted that this study uses only measurements on unprinted uncoated papers. Printed samples, glossier samples and larger specular reflection from rough surfaces will for instance put other demands on the gloss trap, spectral calibration and the linearity.

Table 5. Causes of inter-instrument differences and size of error.

\begin{tabular}{lc}
\hline Cause of error & $\begin{array}{c}\text { Size of error } \\
\Delta E^{*}{ }_{a b}\end{array}$ \\
\hline $\begin{array}{l}\text { Fluorescence and gloss } \\
\text { Using different background for the } R_{0} \\
\text { measurements }\end{array}$ & 7 \\
$\begin{array}{l}\text { Calibrating a } 45 / 0 \text { instrument with a tile } \\
\text { from another instrument of the same type }\end{array}$ & 0.5 \\
$\begin{array}{l}\text { Sample inhomogeneity and small } \\
\text { measurement spot of the } 45 / 0 \text { instrument } \\
\text { Instrument geometry }\end{array}$ & 0.3 \\
\hline
\end{tabular}

Using the KM model for interpretation of measurement data is a part of the problem with inter-instrument differences when calculating a paper's light scattering properties, since it does not recognize the anisotropy (variations in the angular distribution) of the reflected light. An angular resolved model is needed to deal with the situation, and radiative transfer models like DORT2002 can account for geometrical factors in the interpretation of measurement data, which is the key to explain the effect of instrument geometry on inter-instrument differences. This makes it possible to compare different measurement situations objectively, and it recognizes the ubiquitous anisotropy of the reflected light.

A number of results were predicted with DORT2002, and they were confirmed by measurements. The inter-instrument differences when external factors are minimized are small, very close to the instrument precision. Instruments of 45/0 type will detect higher reflectance factor values than $\mathrm{d} / 0$ instruments for highly scattering and nearly opaque samples, while the opposite holds for absorbing or translucent samples. The interinstrument differences are larger for thicker samples, and increase when absorption is negligible and increase further when fillers are present.

Knowledge on how near-surface bulk scattering introduces anisotropy of the reflected light makes it possible to predict how this affects detector readings for different instrument geometries. Instruments of $45 / 0$ type will always have relatively more light reaching a certain depth of the sample, but this will 
affect detector readings differently depending on sample characteristics. The geometry related interinstrument differences can be explained as follows.

- Low absorption and low transmittance results in a higher detector reading for $45 / 0$ than for $\mathrm{d} / 0$ instrument geometry. This is because anisotropy causes relatively more light to reflect in nearnormal angles (towards the detector) with the illumination of the $45 / 0$ geometry (factors 3-4 above dominate, see Fig 7).

- Higher absorption or transmittance results in a lower detector reading for $45 / 0$ than for $\mathrm{d} / 0$ instrument geometry. This is because less light is being reflected altogether for the deeperreaching 45/0 illumination since more will be absorbed or transmitted (factors 1-2 above dominate, see Fig 8).

These results are in accordance with the theoretical predictions and measurements presented in this work.

\section{Conclusions}

A number of paper samples with different properties were examined with the L\&W Elrepho $\mathrm{d} / 0$ and the Spectrolino 45/0 instruments. The external factors that influence the measurements were studied, and a methodology for their minimization was presented. The experimental measurements showed that the geometry related inter-instrument difference when all external factors are minimized is small and of order $0.1 \Delta E_{a b}^{*}$.

It was also observed that the $45 / 0$ instrument detects higher reflectance from paper samples with negligible absorption and transmittance. When there is considerable absorption (dyed samples) or transmittance (thin samples), the $\mathrm{d} / 0$ instrument detects higher reflectance. The physical mechanism behind this behavior was studied and explained, using the DORT2002 radiative transfer model. The measured inter-instrument differences were also compared with the model predictions. The model/measurement agreement was satisfactory as the characteristic behavior was captured in almost all cases. Some variations are to be expected, since the measured differences are close to the instrument precision.

The literature has many statements that $45 / 0$ and $\mathrm{d} / 0$ instruments do differ, and this is usually supported by measurements. Results have not yet been presented that actually explain and quantify the sources of these differences, but this is now done. This work shows the size of a number of external factors, and shows how to minimize them to reduce the inter-instrument differences to a very low level. Further, this work shows how to use the DORT2002 model to predict accurately the remaining differences, which are caused by instrument geometry. This is confirmed by measurements, and the differences are mapped against sample properties. Finally, supported by both simulations and measurements, the physical mechanism behind the inter-instrument differences is explained, showing how absorption and transmittance cause anisotropy in the reflected light and thereby affect detector readings differently. This new understanding is important for facilitating accurate data exchange between the paper and graphic arts industries, but also for interpretation of reflectance measurements in general. This work also illustrates the usefulness of higher order models like DORT2002 for understanding and explaining phenomena not accounted for in simpler models.

\section{Acknowledgements}

This work was financially supported by VINNOVA (The Swedish Governmental Agency for Innovation Systems), the Knowledge Foundation and the Kempe Foundations, which is gratefully acknowledged.

\section{Literature}

Andersson, M. and Norberg, O. (2007): Color Measurements on Prints containing Fluorescent Whitening Agents, Proceedings of Color Imaging XII: Processing, Hardcopy and Applications - IS\&T/SPIE 19th Symposium on Electronic Imaging, San Jose, California.

Berns, Roy S (2000): Billmeyer and Salzman principles of color technology/Roy Berns $3^{\text {rd }}$ ed p. cm. Wiley

Chandrasekhar, S. (1960): Radiative Transfer, Dover, New York.

DIN 5033 (1992): Colorimetry; spectrophotometric method, Deutsches Institut für Normung e. V.

Edström, P. (2004): Comparison of the DORT2002 radiative transfer solution method and the Kubelka-Munk model, Nord. Pulp Paper Res. J., Vol. 19(3), p. 397.

Edström, P. (2005): A Fast and Stable Solution Method for the Radiative Transfer Problem, SIAM Rev. Vol. 47, p. 447.

Edström, P. (2008): A Two-Phase Parameter Estimation Method for Radiative Transfer Problems in Paper Industry Applications, Inverse Probl. Sci. Eng. Vol. 16, p. 927.

Edström, P. (2009): Numerical Performance of Stability Enhancing and Speed Increasing Steps in Radiative Transfer Solution Methods, J. Comput. Appl. Math. Vol. 228, p. 104.

ISO 13655 (1996): Graphic technology - Spectral measurement and colorimetric computation for graphic arts images, International Organization for Standardization, Geneva: Geneva, Switzerland. 
ISO 2469 (1994): Paper, board and pulps - Measurement of diffuse reflectance factor, International Organization for Standardization: Geneva, Switzerland.

ISO 5-4 (1995): Photography, density measurements Geometric conditions for reflection density, International Organization for Standardization: Geneva, Switzerland.

Kubelka, P. and Munk, F. (1931): Ein Beitrag zur Optik der Farbanstriche, Z.Tech. Phys., Vol. 11a, p. 593.

Kubelka, P. (1948): New contributions to the optics of intensely light-scattering materials. Part I, J. Opt. Soc. Am., Vol. 38, p. 448.

Neuman, M. and Edström, P. (2010a): Anisotropic reflectance from turbid media. I. Theory, J. Opt. Soc. Am. A., Vol. 27(5), p. 1032.

Neuman, M. and Edström, P. (2010b): Anisotropic reflectance from turbid media. II. Measurements, J. Opt. Soc. Am. A., Vol. 27(5), p. 1040.

Nicodemus, F.E., Richmond, J.C., Hsia, J.J., Ginsberg, I.W. and Lamperis, T. (1977): Geometrical Considerations and Nomenclature for Reflectance (NBS monograph 160), National Bureau of Standards, Washington.
Pierce, P.E. and Marcus, R.T. (1997): Radiative Transfer Theory Solid Color-Matching Calculations, Color Res. Appl., Vol. 22, p. 72.

Radencic, G., Neumann, E. and Bohan, M. (2008): Spectrophotometer Inter-Instrument Agreement on Standard Reference Materials and Printed Samples, Printing Industries of America/Graphic Arts Technical Foundation, Sewickley, USA.

Seghi, R.R. (1990): Effects of Instrument-measuring Geometry on Colorimetric Assessments of Dental Porcelains, J. Dent. Res., Vol. 69(5), p. 1180.

Spooner, D.L. (1994): Why do measured values taken with different color instruments usually differ?, In: IS\&T and SID's 2nd Color Imaging Conference: Color Science Systems and Applications, Scottsdale, Arizona. pp. 159-164.

Wyzecki, G. and Stiles, W.S. (2000): Color Science, Wiley, New York. 